To be published in Physics Report (Schramm Memorial Volume, 1999)

SUNY-NTG-98-29

\title{
EVOLUTION OF BLACK HOLES IN THE GALAXY
}

\author{
G. E. BROWN and C.-H. LEE \\ Department of Physics and Astronomy, State University of New York \\ Stony Brook, New York 11794-3800, USA \\ HANS A. BETHE \\ Floyd R. Newman Laboratory of Nuclear Studies, Cornell University \\ Ithaca, New York 14853, USA
}

\begin{abstract}
In this article we attempt a consistent calculation of the evolution (and formation rate) of black holes in the Galaxy, especially those in binaries which can be observed by the X-rays from material falling onto them from companion stars. Our chief results come from the bimodal distribution of progenitors of high-mass black holes, stars of ZAMS masses $\sim 20-35 \mathrm{M}_{\odot}$ and $\sim 80-100 \mathrm{M}_{\odot}$. We rely on the evolutionary calculations of Woosley, Langer \& Weaver (1993, 1995) which included mass loss.

In earlier work (Bethe \& Brown 1998a) it was shown that for binaries with masses in the lower of these two intervals RLOF (Roche Lobe Overflow) transfers the $\mathrm{H}$ envelope of the primary to the secondary during the $\mathrm{H}$-shell burning phase (for the bright HMXBs like SMC X-1, Cen X-3, LMC X-4, Vela X-1 and $4 \mathrm{U}$ 1538-52). The "naked" He core of the primary then burns to a much smaller compact object than one "clothed" by the $\mathrm{H}$ envelope (Brown, Weingartner \& Wijers 1996). In this way it was shown that the primaries of the most massive HMXBs 4U 1700-37 and 4U 1223-62 can end up as either a low-mass black hole or neutron star, not much different in mass. In both cases the most probable progenitor mass was $\sim 40 \mathrm{M}_{\odot}$. It was argued that 1700-37 contained a low-mass black hole. Indeed, for some region above $\sim 35 \mathrm{M}_{\odot}$ because of rapid mass loss leading to a W.-R. phase, the He stars burn as "naked", and resulting Fe core masses remain small, $\sim 1.5 \mathrm{M}_{\odot}$ (gravitational).

Recently Bethe \& Brown (1998b) showed that very massive progenitors of ZAMS masses $\sim 80-100 \mathrm{M}_{\odot}$ were needed to evolve high-mass black holes in binaries such as Cyg X-1, with massive companion. These are important for
\end{abstract}


LIGO and LISA which will detect gravitational waves from mergers. Although there are few of them, their mergers can be seen to higher distances because of their greater mass.

The most copious high-mass black-holes of masses $\sim 6-7 \mathrm{M}_{\odot}$ have been found in the transient sources such as A0620. These have low-mass companions, predominantly of $\lesssim 1 \mathrm{M}_{\odot}$, such as $\mathrm{K}$ - or M-stars. In the progenitor binaries the mass ratios must have been tiny, say $q \sim 1 / 25$. Normally such small ratios are thought to be rare; e.g. in binary evolution the companion distribution is often taken as $d q$, implying an extremely low probability of such a binary, but there are hardly grounds for this when $q$ is so small.

In this note we show that the reason for this small $q$-value lies in the common envelope evolution of the binary. The smaller the companion mass, the greater the radius $R_{g}$ the giant must reach (in the supergiant stage) before its envelope meets the companion. This results because a low-mass companion can furnish only enough energy by tightening its orbit to expel a loosely bound envelope. The binding of the latter goes as $R_{g}^{-1}$.

In other words, in order to force the helium core to be "clothed" while it burns, and to get the main sequence star close to it, we need a small companion mass. We believe this is why $\mathrm{K}$ - and $\mathrm{M}$-star companions are favored.

Once exposed, following the loss of the hydrogen envelope, the helium stars will lose mass rapidly by wind. The higher mass black holes from the ZAMS interval $\sim 20-35 \mathrm{M}_{\odot}$ lose more mass by wind than the lower mass ones, so the trend is to a clustering about $\sim 6-7 \mathrm{M}_{\odot}$.

Subject headings: black hole physics — X-rays: bursts — stars: binaries

\section{INTRODUCTION}

In this note we attempt to make a consistent picture of the evolution of high-mass black holes, those with mass $M_{B H} \gtrsim 3 \mathrm{M}_{\odot}$, where this limit was thought for many years to be a reasonable upper limit for neutron star masses. In particular, we shall differentiate them from the low-mass black holes $M_{L M B H} \sim 1.5-3 \mathrm{M}_{\odot}$ of Brown \& Bethe (1994). We take some care in this differentiation, because it is clear from the literature that the Brown-Bethe scenario has not been widely accepted.

Such a consistent picture is necessary in order to give reasonable predictions for the mergers of high-mass black holes with companion neutron stars. Generally these mergers, 
or the merger of two high-mass black holes in a binary are predicted to give the strongest signals for gravitational wave detectors, such as LIGO. Consequently, it is imperative to lay out the best theoretical predictions before LIGO reaches sufficient sensitivities to detect mergers of binaries of compact objects.

Black-hole, neutron star binaries are also considered (Rees 1997) as "best buy" progenitors for gamma-ray bursters. For black holes more massive than $5-6 \mathrm{M}_{\odot}$ the neutron star would be swallowed before disruption. The low-mass black-hole, neutron-star binaries recently evolved by Bethe \& Brown (1998a) in which the black-hole mass is typically $\sim 2.4 \mathrm{M}_{\odot}$, and the neutron-star mass $\sim 1.4 \mathrm{M}_{\odot}$, seem to offer good candidates for GRBs.

Another class of black-hole, neutron-star binaries is offered (Brown 1997) by the binaries evolved from double helium stars in which the pulsar originating from the explosion of the first helium star evolves into a black hole by accretion from the companion helium star as it expands in He shell burning. This occurs for ZAMS masses $\lesssim 15 \mathrm{M}_{\odot}$. The mass of the black hole depends on the amount of mass accreted from the evolving He envelope of the companion, probably enough to send it into a black hole, but not much more.

In the disk of the Galaxy only one HMXB is known, Cyg X-1, although two are known in the LMC: LMC X-1 and LMC X-3. X-rays of more than $3 \mathrm{keV}$ energy should be seen throughout the Galaxy, so why Cyg X-1 at a distance of only $2.5 k p c$ is the only HMXB observed in the disk is a puzzle. Perhaps Cyg X-1 like objects exist at greater distances, where the O-star companion is difficult to identify so the X-rays are not associated with a binary.

Most high-mass black holes observed are in the transient sources (which flare up from time to time), seen as LMXB's; i.e., they have predominantly low-mass main sequence companions of mass $\lesssim 1 \mathrm{M}_{\odot}$. Many of these have been discovered recently with the Compton Gamma-ray Observatory and advances in optical astronomy which make possible the spectroscopic determination of the radial velocity curves of many of their faint companions. These transient sources generally contain high-mass black holes of mass $\sim 6-7 \mathrm{M}_{\odot}($ Wijers 1996; Bailyn et al. 1998).

In the evolution of HMXB's in binaries, where the H-envelope of the black-hole progenitor is transferred in RLOF either before or early in the core He burning stage of the progenitor, the "naked" He star evolves to a substantially less massive compact object than had it been clothed. The reason is that convective carbon burning is essentially never skipped in the "naked" He stars, and this leads to substantially lower mass Fe cores than in single stars where the He envelope remains "clothed" as we discuss in Section 3. Thus, the 
lower limit on the ZAMS mass of the progenitor of a HMXB is $\sim 80 \mathrm{M}_{\odot}$ (Bethe \& Brown 1998b). This high limit partially explains the paucity of Cyg X-1 like objects. However, in the case of the transient sources in which the main sequence companion has mass $\lesssim 1 \mathrm{M}_{\odot}$, the removal of the H-envelope in the primary occurs only following He core burning in the primary. If the main sequence companion is met by the envelope earlier in its evolution, the former will generally spiral into the primary. If its He core burning is completed before the H-envelope has evolved to the radius of the main sequence companion, the primary will evolve as a single star. In our case the main sequence star has to be $\sim 2$ A.U. distant from the primary in order to survive spiral-in, so the He core burning is finished. We have evolutionary results for compact objects from Woosley, Langer \& Weaver (1995) and Woosley \& Weaver (1995) for the burning of either fully "naked" or fully "clothed" He stars; i.e., either with no H-envelope or with the full one. The clothed ones burn as single stars. As we show in Fig. 1 from Brown, Weingartner \& Wijers (1996), there is a great difference between the compact objects resulting from single stars or from "naked" He cores. In a wide range from ZAMS $\sim 20-25 \mathrm{M}_{\odot}$ to $\sim 35-40 \mathrm{M}_{\odot}$ the compact object from the single star exceeds $1.7-1.8 \mathrm{M}_{\odot}$, sufficient for the star to evolve into a high-mass black hole, swallowing all of its He core. The compact object from the "naked" He core tends to be $\sim 1.5 \mathrm{M}_{\odot}$ in the higher mass range of Fig. 1 and will end up either as a neutron star or low-mass black hole.

We can identify the transition from single stars which evolve into low-mass black holes and high-mass ones, at least roughly, with the large jump in compact core masses at the change over from convective to radiative carbon burning (Timmes, Woosley \& Weaver 1996).

Stars with ZAMS masses $\gtrsim 40 \mathrm{M}_{\odot}$ lose their masses by strong winds, whether in binaries or not, and become Wolf-Rayet stars. In an earlier paper Woosley, Langer \& Weaver (1993) investigated ZAMS masses of 35, 40, 60 and $85 \mathrm{M}_{\odot}$. In those up through $60 \mathrm{M}_{\odot}$ the hydrogen envelope was blown off early enough for the He cores to evolve as naked ones and compact cores were $\gtrsim 1.5 \mathrm{M}_{\odot}$ (gravitational). In fact, with inclusion of extensive mass loss the lower lines in Fig. \&, which heads just above $1.5 \mathrm{M}_{\odot}$ for the higher ZAMS masses, gave the WLW (Woosley, Langer \& Weaver 1993) correspondence of Fe core mass to ZAMS mass for single stars of masses $35-60 \mathrm{M}_{\odot}$. Thus, rapid mass loss by wind in this mass region which removes the $\mathrm{H}$-envelope before appreciable He core burning leaves a He core which burns as a "naked" one. (In the case of the massive stars the relation shown in Fig. 11 between Fe core mass and He core mass no longer holds because of large wind losses in the latter.)

Basically, as we outline in the next section, either most of the He envelope collapses 
inwards as the compact object goes into a black hole, or there is an explosion with return of matter to the galaxy and then the compact object evolves into a low-mass black hole or a neutron star (Brown \& Bethe 1994). Thus, a bimodal distribution of black holes results, the high-mass ones which result from collapse of massive He cores, or the low-mass ones, which may not be much more massive than a neutron star. In the former case, the He cores are initially $\sim 7-10 \mathrm{M}_{\odot}$, but by the time of collapse will have diminished somewhat because of the large mass loss rate of He star. We should attempt some more precise estimates later. In the latter case, the black-hole mass need not exceed the maximum neutron star mass $M_{N S}(\max ) \sim 1.5 \mathrm{M}_{\odot}$ (Brown \& Bethe 1994) by much, but can also be somewhat more massive. In particular, in common envelope evolution, it could have accreted $\sim 1 \mathrm{M}_{\odot}$ (Bethe \& Brown 1998a). In the case of 1700-37 which has not gone through common envelope evolution the observed mass is $1.8 \pm 0.4 \mathrm{M}_{\odot}$, difficult to measure to higher accuracy because of the low velocity of the high-mass companion.

In the case of the He cores in high-mass primaries accompanied by a low-mass secondary, the entire He core will collapse into a high-mass black hole if the $\mathrm{H}$ envelope has been removed only late in the He core burning.

In section 2 we shall delineate high-mass black holes from low-mass ones and give our estimates of core masses necessary to produce each of these, as well as reviewing the maximum mass that a neutron star can have. In section 3 we discuss in detail the difference in evolution between "clothed" and "naked" He cores. In section 4 we list our assumptions for the evolution of transient sources, as well as calculating this evolution. In section 5 we estimate the birth rate of transient sources and compare it with that of low-mass X-ray binaries. In section 6 we discuss He-star winds with the aim of estimating the masses of the high-mass black holes in transient sources. In section 7 we give an overview of high-mass black holes and in section 8 we state our conclusions.

\section{THE COMPACT STAR}

Thorsson, Prakash \& Lattimer (1994) and Brown \& Bethe (1994) have studied the compact core after the collapse of a supernova, assuming reasonable interactions between hadrons. Initially, the core consists of neutrons, protons and electrons and a few neutrinos. It has been called a proto-neutron star. It is stabilized against gravity by the pressure of the Fermi gases of nucleons and leptons, provided its mass is less than a limiting mass $M_{P C}$ (proto-compact) of $\sim 1.8 \mathrm{M}_{\odot}$.

If the assembled core mass is greater than $M_{P C}$ there is no stability and no bounce; the 
core collapses immediately into a black hole. It is reasonable to take the core mass to be equal to the mass of the Fe core in the pre-supernova, and we shall make this assumption, although small corrections for fallback in the later supernova explosion can be made as in Brown, Weingartner \& Wijers (1996). If the center collapses into a black hole, the outer part of the star has no support other than centrifugal force from angular momentum and will also collapse. Our neglect of effects from angular momentum is admittedly naive. We then get a massive black hole containing the entire mass of the presupernova star, perhaps of order $\lesssim 10 \mathrm{M}_{\odot}$. (At least in binaries the companion star will have removed the hydrogen envelope by either Roche Lobe Overflow or by common envelope evolution, depending on the mass of the companion, and wind will diminish the He core before the SN explosion.)

If the mass of the core is less than $M_{P C}$, the electrons will be captured by protons

$$
p+e^{-} \rightarrow n+\nu
$$

and the neutrinos will diffuse out of the core. This process takes of order of 10 seconds, as has been shown by the duration of the neutrino signal from SN1987A. The result is a neutron star, with a small concentration of protons and electrons. The Fermi pressures of the core are chiefly from the nucleons, with small correction from the electron. On the other hand the nucleon energy is increased by the symmetry energy; i.e., by the fact that we now have nearly pure neutrons instead of an approximately equal number of neutrons and protons. Thorsson et al. (1994) have calculated that the maximum mass of the neutron star $M_{N S}$ is still about $1.8 \mathrm{M}_{\odot}$; i.e., the symmetry energy compensates the loss of the Fermi energy of the leptons. Corrections for thermal pressure are small (Prakash et al. 1997).

The important fact is that the ten seconds of neutrino diffusion from the core give ample time for the development of a shock which expels most of the mass of the progenitor star.

But this is not the end of the story. The neutrons can convert into protons plus $K^{-}$ mesons,

$$
n \rightarrow p+K^{-} .
$$

Since the density at the center of the neutron star is very high, the energy of the $K^{-}$is very low, as confirmed by Li, Lee \& Brown (1997) using experimental data. By this conversion the nucleons can again become half neutrons and half protons, thereby saving the symmetry energy needed for pure neutron matter. The $K^{-}$, which are bosons, will condense, saving the kinetic energy of the electrons they replace. The reaction (2.2) will be slow, since it is preceded by

$$
e^{-} \rightarrow K^{-}+\nu
$$


with the reaction (2.2) following as it becomes energetically advantageous to replace the fermionic electrons by the bosonic $K^{-}$'s at higher densities. Initially the neutrino states in the neutron star are filled up to the neutrino chemical potential with trapped neutrinos, and it takes some seconds for them leave the star. These must leave before new neutrinos can be formed from the process (2.3). Thorsson et al. (1994) have calculated that the maximum mass of a star in which reaction (2.2) has gone to completion is

$$
M_{N P} \simeq 1.5 \mathrm{M}_{\odot},
$$

where the lower suffix $N P$ denotes their nearly equal content of neutrons and protons, although we continue to use the usual name "neutron star". This is the maximum mass of neutron stars, which is to be compared with the masses determined in binaries. The masses of 19 neutron star masses determined in radio pulsars (Thorssett \& Chakrabarty 1998) are consistent with this maximum mass.

The core mass $M_{C}$ formed by the collapse of a supernova must therefore be compared to the two limiting masses, $M_{P C}$ and $M_{N P}$. If

$$
\text { (I) } M_{C}>M_{P C}
$$

we get a high mass black hole. If

$$
\text { (II) } M_{P C}>M_{C}>M_{N P}
$$

we get a low mass black hole, of mass $M_{C}$. Only if

$$
\text { (III) } M_{C}<M_{N P}
$$

do we get a neutron (more precisely, "nucleon") star from the SN. Only in this case can we observe a pulsar. In cases (II) and (III) we can see a supernova display. In case (I) we receive only initial neutrinos from electrons captured in the collapse before $M_{C}$ becomes greater than $M_{P C}$ but no light would reach us.

We tentatively choose the lower limit of ZAMS mass for making MBHs with massive companion to be $80 \mathrm{M}_{\odot}$. On the other hand it is believed that ZAMS stars above $100 \mathrm{M}_{\odot}$ do not exist, because of excessive formation of electron pairs. So we assume that the range of 80 to $100 \mathrm{M}_{\odot}$ is available.

Following our discussion of sizes of compact objects which evolve from various ZAMS mass single stars and stars in binaries in which the He cores evolve as "naked", we give in Table 2 our projected relation between ZAMS mass and type of compact object. 


\section{CONVECTIVE CARBON BURNING (AND ITS DISAPPEARANCE)}

The convective carbon burning phase is extremely important in supernova evolution, when it occurs, because this is the first phase in which a large amount of entropy can be carried off in $\nu \bar{\nu}$-pair emission, especially if this phase is of long duration. The lower the ${ }^{12} C(\alpha, \gamma){ }^{16} O$ cross section, the higher the temperature of the ${ }^{12} C$ burning, because other channels such as ${ }^{12} C+{ }^{12} C$ going into various products require a much higher temperature $\sim 80 \mathrm{keV}$ than that of the direct ${ }^{16} \mathrm{O}$ production through $\alpha$-capture. Thus, with the relatively low ${ }^{12} C(\alpha, \gamma){ }^{16} O$ rates determined both directly from nuclear reactions and from nucleosynthesis by Weaver \& Woosley (1993), the entropy carried off during ${ }^{12} C$ burning in the stars of ZAMS mass $\leq 18 \mathrm{M}_{\odot}$ is substantial. The result is rather low mass Fe cores for these stars, which can evolve into neutron stars. Note that in the literature earlier than Weaver \& Woosley (1993) often large ${ }^{12} C(\alpha, \gamma){ }^{16} O$ rates were used, so that the ${ }^{12} C$ was converted into oxygen and the convective burning did not have time to be effective. Thus its role was not widely appreciated.

Of particular importance is the ZAMS mass at which the convective carbon burning is skipped. In the Woosley \& Weaver (1995) calculations this occurs at ZAMS mass $19 \mathrm{M}_{\odot}$

but with a slightly lower ${ }^{12} C(\alpha, \gamma){ }^{16} O$ rate it might come at $20 \mathrm{M}_{\odot}$ or higher (Brown 1997). As the progenitor mass increases, it follows from general polytropic arguments that the entropy at a given burning stage increases. At the higher entropies of the more massive stars the density at which burning occurs is lower, because the temperature is almost fixed for a given fuel. Lower densities decrease the rate of the triple- $\alpha$ process which produces ${ }^{12} \mathrm{C}$ relative to the two-body ${ }^{12} C(\alpha, \gamma){ }^{16} \mathrm{O}$ which produces oxygen. Therefore, at the higher entropies in the more massive stars the ratio of ${ }^{12} \mathrm{C}$ to ${ }^{16} \mathrm{O}$ at the end of He burning is lower. At the above crossover, the star skips the long convective carbon burning and goes on to the much shorter oxygen burning. Since neutrino cooling during the long carbon-burning phase gets rid of a lot of entropy of the core, skipping this phase leaves the core entropy higher and the final Chandrasekhar core fatter.

In Fig. 1 the large jump in compact object mass in single stars at ZAMS mass $\sim 19 \mathrm{M}_{\odot}$ is clearly seen. From our discussion in the last session we see that this is just about at the point where our proto-compact mass $M_{P C}$ goes above $\sim 1.8 \mathrm{M}_{\odot}$ and, therefore, above this mass one would expect single stars to go into high-mass black holes. Arguments have been given that SN (1987A) with progenitor ZAMS mass of $\sim 18 \mathrm{M}_{\odot}$ evolved into a low-mass black hole (Brown \& Bethe 1994). We believe from our above arguments and Fig. 1 that just above the ZAMS mass of $\sim 18 \mathrm{M}_{\odot}$, single stars go into high-mass black holes without return of matter to the Galaxy. Thus, the region of masses for low-mass black hole formation in single stars is narrow, say $\sim 18-20 \mathrm{M}_{\odot}$. Taking Fig. 1 seriously, the precise 
mass limit $M_{P C}$ is not clear, but certainly in the range of $\sim 20 \mathrm{M}_{\odot}$

Thus far our discussion has been chiefly about single stars, in which the He burns "clothed" by a hydrogen envelope. In this case the convective helium core grows in stars of constant mass as time passes. In the "naked" He cores, in which the H envelope has been lifted off in RLOF or driven off by wind either before or early in the He burning the temperature will be slightly lower, because the insulating layer is gone, and this decreases the rate of ${ }^{12} \mathrm{C}$ burning. However, the main effect results from mass loss by wind, the helium core shrinking when substantial mass loss takes place. Because of mass loss, naked He cores of massive stars of ZAMS mass $>20 \mathrm{M}_{\odot}$, are similar to those of clothed less massive stars, $\sim 15 \mathrm{M}_{\odot}$, so it is not surprising that their carbon abundance is large. In fact, even for the naked $20 \mathrm{M}_{\odot}$ He core, corresponding to ZAMS $\sim 50 \mathrm{M}_{\odot}$ from Fig. $\mathbb{1}$, the central carbon abundance was $\sim 33 \%$ at the end of He core burning (Woosley \& Weaver 1995) whereas only $\sim 15 \%$ is necessary for convective carbon burning (Weaver \& Woosley 1993). For lower mass He stars the ${ }^{12} \mathrm{C}$ abundance was, of course, larger. Thus, in the range of ZAMS masses up to $\geq 50 \mathrm{M}_{\odot}$ (and beyond), if the $\mathrm{H}$ envelope is lifted off early in the core He burning phase, the convective carbon burning will take place throughout the He core burning. By ZAMS mass $\sim 40 \mathrm{M}_{\odot}$ where stars evolve into W.-R. stars almost independently of possible companion, the ultimate fate of the compact core is uncertain: Brown, Weingartner \& Wijers (1996) suggest that 1223-62, with progenitor of mass $\sim 40 \mathrm{M}_{\odot}$ went into a neutron star, whereas 1700-37, with similar mass progenitor, into a low-mass black hole. This can be understood from Fig. 1, where the compact core would be $\sim 1.5 \mathrm{M}_{\odot}$ (gravitational) because this is just the mass at our dividing line between neutron stars and low mass black holes. For ZAMS masses $\sim 40 \mathrm{M}_{\odot}$ the outcome could be either. It is, however, clear that when RLOF occurs early in the evolution, say during $\mathrm{H}$ shell burning, of a binary of two massive stars, the progenitor of the high-mass black hole must be much greater, possibly the $\sim 80 \mathrm{M}_{\odot}$ suggested by Bethe \& Brown (1998b). As discussed by Bethe \& Brown, even such massive stars seem to evolve into high mass black holes in binaries only because of a substantial WNL phase; i.e., the He core is covered by some $\mathrm{H}$ envelope for a substantial part of the He core burning.

\section{ASSUMPTIONS}

We make roughly the same assumptions as Bethe \& Brown (1998a,b), our massive star $M_{A}$ lying in mass somewhere in between the O,B star progenitors of binary neutron stars and the progenitor of the massive black hole in Cyg X-1. Our low-mass companion is a 
main sequence star of mass $\sim 1 \mathrm{M}_{\odot}$. Thus, the ratio

$$
q=\frac{M_{B 0}}{M_{A 0}}
$$

is very small, and there will be great uncertainty in the initial number of binaries for such a small $q \sim 1 / 25$. We take the distribution as $d q$. The distribution in $q$ is unknown for such low-mass companions as are involved here, but our results will show that the flat distribution in $q$ is not unreasonable. We assume $\log a$, where $a$ is the semi-major axis of the orbit, to be uniformly distributed, over a total logarithmic interval of 7 . Thus, the fraction of binaries in a given interval of $\ln a$ is

$$
d \phi=\frac{d(\ln a)}{7}
$$

We take $\alpha$ the supernova rate to be $\sim 0.015$ per galaxy per year (Cappellaro et al. 1997), somewhat smaller than the 0.025 assumed by Bethe \& Brown (1998a). The rate of supernovae $(\mathrm{SN})$ is the same as the rate of birth of massive stars $M>10 \mathrm{M}_{\odot}$, so

$$
d \alpha=\alpha n\left(\frac{M}{10 \mathrm{M}_{\odot}}\right)^{-n} \frac{d M}{M}
$$

with $n$ the Salpeter exponent which we take to be 1.5. We furthermore assume a binarity of 0.5 . Once again, for our very small $q$ values this is uncertain.

From Fig. 11 we take the single stars which have masses great enough to go into a high-mass black hole (i.e., the compact core mass is $\gtrsim 1.8 \mathrm{M}_{\odot}$ ) to lie in the interval $20-35 \mathrm{M}_{\odot}$, with $25 \mathrm{M}_{\odot}$ typical. Stars in the neighborhood of ZAMS mass $40 \mathrm{M}_{\odot}$ and above suffer extensive mass loss and go quickly into a Wolf-Rayet stage. Their He cores, at least up to $\sim 85 \mathrm{M}_{\odot}$ (Woosley, Langer \& Weaver 1993) evolve as naked, independent of whether they have a companion or not. From Fig. 1 it can be seen that they will evolve into compact core masses of only $\sim 1.5 \mathrm{M}_{\odot}$, and end up as either a low-mass black hole or a neutron star. The ZAMS $85 \mathrm{M}_{\odot}$ star of Woosley, Langer \& Weaver (1993) evolved as partially clothed, and would go into a high-mass black hole. Bethe \& Brown (1998b) took the limit as $80 \mathrm{M}_{\odot}$.

We thus evolve a ZAMS $25 \mathrm{M}_{\odot}$ with companion $\sim 1 \mathrm{M}_{\odot}$ main sequence star as typical progenitor of the transient X-ray sources. The common envelope evolution can be done as in Bethe \& Brown (1998a). With $M_{B_{i}}=25 \mathrm{M}_{\odot}$ and neglect of the accretion onto the main sequence mass $M_{A}$, B from Bethe \& Brown we find

$$
\left(\frac{Y_{f}}{Y_{i}}\right)^{1.2}=\frac{1.2}{\alpha_{c e}} \frac{M_{B_{i}}}{M_{A}}
$$


where $Y=M_{B} / a$. Here the coefficient of dynamical friction $c_{d}$ was taken to be 6 , in the range of the 6-8 for supersonic flow with Mach number 3-10 (Ruffert 1994; Ruffert \& Arnett 1994). The result is relatively insensitive to $c_{d}$, the exponent 1.2 resulting from $1+1 /\left(c_{d}-1\right)$.

Thus, in our case

$$
\frac{Y_{f}}{Y_{i}}=17\left(\frac{\alpha_{c e} M_{A}}{M_{\odot}}\right)^{-0.83}=30\left(\frac{0.5}{\alpha_{c e}} \frac{M_{\odot}}{M_{A}}\right)^{0.83}
$$

We expect $\alpha_{c e} \simeq 0.5$, under the assumption that the kinetic energy of the expelled envelope is equal to that it originally possessed in the massive star, but it could be smaller. From this we obtain

$$
\frac{a_{i}}{a_{f}}=\frac{M_{B_{i}} Y_{f}}{M_{B_{f}} Y_{i}}=90\left(\frac{0.5}{\alpha_{c e}} \frac{M_{\odot}}{M_{A}}\right)^{0.83},
$$

where we have taken the He star mass $M_{B_{f}}$ to be $1 / 3$ of $M_{B_{i}}$. In order to survive spiral-in, the final separation $a_{f}$ must be sufficient so that the main sequence star lies inside its Roche Lobe, about $0.2 a_{f}$. This sets $a_{f} \sim 5 R_{\odot}=3.5 \times 10^{11} \mathrm{~cm}$ and

$$
a_{i}=3.15\left(\frac{0.5}{\alpha_{c e}} \frac{M_{\odot}}{M_{A}}\right)^{0.83} \times 10^{13} \mathrm{~cm} .
$$

$\sim 2$ A.U.. Notice that decreasing $\alpha_{c e} M_{A}$ will increase $a_{i}$. However, decreasing $M_{A}$ alone will not give a large change, because with the smaller solar radius the minimum $a_{f}$ will decrease nearly proportionately. Note that neglect of accretion onto the main sequence star would change the exponent 0.83 to unity, so accretion is unimportant except in increasing the final mass.

Now a ZAMS $25 \mathrm{M}_{\odot}$ star ends up at radius $6.7 \times 10^{13} \mathrm{~cm}\left(\sim 2 a_{i}\right)$ following He core burning (Weaver, Zimmerman \&Woosley 1978). Thus the interval between $a_{i}$ and $6.7 \times 10^{13}$ $\mathrm{cm}$ is available for spiral-in without merger so that a fraction

$$
\frac{1}{7} \ln \left(\frac{6.7}{3.15\left(\frac{0.5}{\alpha_{c e}} \frac{M_{\odot}}{M_{A}}\right)^{0.83}}\right) \simeq 0.11
$$

of the binaries survive spiral-in, but are close enough so that the main sequence star is encountered by the evolving $H$ envelope of the massive star. Note that the survivors all come from the supergiant region following that of He core burning, the evolution in the earlier $\mathrm{H}$ shell burning (which takes about the same time as the He core burning) reaching out to less than $10^{13} \mathrm{~cm}$. The He core burning will be completed before the supergiant has moved out to $\sim 2$ A.U., so any binaries which survive spiral-in will have He cores which burn as "clothed", namely as in single stars. 


\section{BIRTH RATE OF TRANSIENT SOURCES}

Given our assumptions in Section 2, the fraction of supernovas which arise from ZAMS stars between 20 and $35 \mathrm{M}_{\odot}$ is

$$
\frac{1}{2^{3 / 2}}-\frac{1}{3.5^{3 / 2}}=0.20
$$

where we have assumed the mass $10 \mathrm{M}_{\odot}$ is necessary for a star to go supernova. Our assumption that the binary distribution is as $d q$ is arbitrary, and gives us a factor $1 / 25$ for a $1 \mathrm{M}_{\odot}$ companion. Note, however, that had we included higher mass companions, the change in the final projected number of transient sources would not be order of magnitude, because the hydrogen burning time goes inversely as mass squared. Thus, for supernova rate 1.5 per century, our birth rate for transient sources in the Galaxy is (taking the companion mass to be $1 \mathrm{M}_{\odot}$ )

$$
1.5 \times 10^{-2} \times 0.5 \times 0.11 \times 0.20 \times 0.04 \simeq 6 \times 10^{-6} \mathrm{yr}^{-1}
$$

where 0.5 is the assumed binarity, 0.11 comes from eq. 4.8). We comment later on the final (most uncertain) factor 0.04 which results from a distribution flat in $q$ and an assumed $1 \mathrm{M}_{\odot}$ companion star.

In order to estimate the number of transient sources in the Galaxy, we should know the time over which a main sequence star of mass $\sim 1 \mathrm{M}_{\odot}$ can transfer mass to a more massive companion. Van den Heuvel (1994, p. 316) estimates a mass transfer rate of $\sim 10^{-10}$ to $10^{-9} \mathrm{M}_{\odot} \mathrm{yr}^{-1}$. One would expect the greater rate to apply to the two systems that have subgiant donors (V404 Cyg and XN Sco94; Wijers 1996). Given the birth rate eq. (5.2) this would give $\gtrsim 4000$ transient sources in the Galaxy where we have taken the unevolved main sequence stars to be $3 / 4$ of the total. Wijers estimates $\sim 3000$, but considers this to be a lower limit.

If we assume that ZAMS masses $10 \sim 18 \mathrm{M}_{\odot}$ evolve into a neutron star we should have $\sim 3$ times more neutron stars than high-mass black holes (see eq. (5.1)). The upper limit follows from our belief that SN (1987A) with progenitor $\sim 18 \mathrm{M}_{\odot}$ went into a low-mass black hole, following the scenario of Brown \& Bethe (1994). On the basis of a Monte Carlo calculation using the kick velocities of Cordes \& Chernoff (1997) we find that $\sim 1 / 2$ of the He-star, low-mass main sequence star (with $M=1 \mathrm{M}_{\odot}$ ) will be disrupted in the explosion. Thus, we find only slightly more LMXB's with neutron stars as with black holes although the numbers could be equal to within our accuracy. Note that our relatively large number of transient black hole sources results from the bimodality of our mass function for evolving into high-mass black holes; i.e., $20-35 \mathrm{M}_{\odot}$ and $80-100 \mathrm{M}_{\odot}$ (Bethe \& Brown 
1998b). With a single mass limit of $40 \mathrm{M}_{\odot}$, Portegies Zwart, Verbunt \& Ergma (1997) were short by at least two orders of magnitude in evolving the black-hole transient sources. Portegies Zwart (1998) has kindly redone their calculations using our bimodal distribution for high-mass black hole evolution, with a Salpeter exponent 1.35. He obtains a birth rate of $2.6 \times 10^{-6} \mathrm{yr}^{-1}$ for transient sources with a high-mass black hole and $9.0 \times 10^{-6} \mathrm{yr}^{-1}$ for transient sources with a neutron star. The latter should be decreased by a factor $\sim 2$ to take into account the disruption of the He-star, main-sequence star binary in the explosion. In this explosion the neutron star gets a kick velocity (whereas the high-mass black holes do not in the evolution of the transient black-hole sources).

\section{ESTIMATED MASSES OF THE BLACK HOLES IN TRANSIENT SOURCES}

In order to estimate the final mass of the He star before explosion, we choose a ZAMS $20 \mathrm{M}_{\odot}$ star. This is because the mass loss rates used in calculations go as the 2.5 or 2.6 power of the mass, and the more massive stars will quickly lose mass down to final values near to that for a lower-mass star. Empirically most W.-R. stars are found to have masses $\sim 10 \mathrm{M}_{\odot}$, but there is a strong bias against observing lower-mass W.-R.'s because observation is usually in terms of wind.

For an initial $7 \mathrm{M}_{\odot}$ He star, Woosley, Langer \& Weaver (1995) find a final mass of $3.2 \mathrm{M}_{\odot}$. However, the mass loss rates estimated from the winds are measured chiefly via free-free scattering and depend quadratically on the density. Measurements from the polarization (St. Louis et al. 1993) which depend linearly on the density, give a mass loss rate of $\dot{M}=0.75 \times 10^{-5} \mathrm{M}_{\odot} \mathrm{yr}^{-1}$, for V444 Cygni, an $\sim 10 \mathrm{M}_{\odot}$ Wolf-Rayet star. This rate is $\sim 40 \%$ of that used by Woosley, Langer \& Weaver (1995). Using the lower rate, one ends up with $M_{f} \sim 5.5 \mathrm{M}_{\odot}$ for an $M_{i}=7 \mathrm{M}_{\odot}$ He star. The $20 \mathrm{M}_{\odot}$ is at the lower limit of our massive black hole progenitor, so we would estimate their masses as $\gtrsim 5.5 \mathrm{M}_{\odot}$. Measured mass functions, which give a lower limit on the black hole mass are given in Table 1. Only GRO J0422+32 and 4U 1543-47 out of the measured mass function are $\lesssim 3 \mathrm{M}_{\odot}$. Results of Callanan et al. (1996) indicate that the angle $i$ between the orbital plane and the plane of the sky for GRO J0422+32 is $i<45^{\circ}$, and recent analysis by Orosz et al. (1998) indicate that the angle $i$ for $4 \mathrm{U} 1543-47$ is $20^{\circ}<i<40^{\circ}$. So both GRO J0422+32 and $4 \mathrm{U} 1543-47$ also contain high-mass black holes. 


\section{HIGH MASS X-RAY BINARIES}

We return to a brief discussion of Cyg X-1-like objects, which will give strong gravitational waves when the black hole merges with the compact object resulting from the companion star. While high-mass black hole progenitors have a bimodal distribution, those in binaries can come from ZAMS $>80 \mathrm{M}_{\odot}$ as Bethe \& Brown (1998b) have discussed. The fraction of binaries which go supernova in which one star is in the interval $80-100 \mathrm{M}_{\odot}$ was found to be $1.26 \times 10^{-2}$. Since they can have a companion of almost any mass, Bethe \& Brown multiplied by $1-q \simeq 0.9$ taking the flat distribution in $q$, in order to obtain the number of binaries in which both stars go supernova. The $1.26 \times 10^{-2}$ is to be compared with the much larger proportion of single stars with ZAMS masses $20-35 \mathrm{M}_{\odot}$ that go into high-mass black holes, the 0.20 of eq. (5.1). But the latter, when multiplied by our $q \sim 1 / 25$ for binarity, gives $0.8 \times 10^{-2}$, not very different from the $1.13 \times 10^{-2}$ for high-mass black holes in a binary with a high-mass companion.

It has often been suggested that the distribution of binaries in $q$ is peaked about equal $q$, although much of this may be a selection effect. The assumptions of a distribution flat in $q$ take roughly into account the very many more lower mass stars, and may not be unreasonable. Bailyn et al. (1998), on the basis of a Bayesian analysis, suggest that V404 Cyg has a mass significantly greater than the others of the transient sources. Leaving out V404 Cyg the maximum likelihood limits of the transient sources become close $\left(m_{l}=6.91\right.$ and $m_{u}=6.97 \mathrm{M}_{\odot}$ ). This is not inconsistent with the $\gtrsim 5.5 \mathrm{M}_{\odot}$ we estimated, based on the He core mass of the least massive star in our interval; e.g., $7 \mathrm{M}_{\odot}$ would be about right for a ZAMS $25 \mathrm{M}_{\odot}$ star.

The higher V404 Cyg mass suggests that the high mass black hole came from the same mass region $>80 \mathrm{M}_{\odot}$ ZAMS used by Bethe \& Brown (1998b) to make the black hole in Cyg $\mathrm{X}-1$. For a distribution flat in $q$ this would occur only about $q$ as often as an $\mathrm{O}$ or B-star companion (taking the black hole in Cyg X-1 to come from a $90 \mathrm{M}_{\odot}$ star). However, the

longer lifetime of the low-mass companion compensates insofar as the observable number of binaries is concerned.

\section{CONCLUSION}

We have been able to develop a scenario for black-hole evolution consistent with observations. We find that massive black holes evolve from stars with ZAMS masses $\sim 20-35 \mathrm{M}_{\odot}$ and $\sim 80-100 \mathrm{M}_{\odot}$. This bimodal distribution is based on the evolution of massive stars including mass loss by Woosley, Langer \& Weaver (1993). Using a 1995 
calculation for "naked" He stars by the same authors, Brown, Weingartner \& Wijers (1996) were able to show why compact objects in the known HMXBs were either neutron stars or low-mass black holes. The HMXB 1700-37 was a good candidate for the latter. These authors showed that ZAMS masses up to $\sim 40 \mathrm{M}_{\odot}$ could give these small compact cores of mass $\sim 1.5 \mathrm{M}_{\odot}$. The key point was that "naked" He stars, produced in binaries by RLOF, burned to much smaller compact objects than the "clothed" ones in single stars. Woosley, Langer \& Weaver (1993) showed that if stars of ZAMS mass $\gtrsim 35 \mathrm{M}_{\odot}$ lost their $\mathrm{H}$ envelope early in the He burning by wind, that small mass compact cores could result in single stars up to much higher masses, $\sim 80 \mathrm{M}_{\odot}$; i.e., the continuation of the lower curve in our Fig. 1 does not exceed our $M_{P C} \sim 1.8 \mathrm{M}_{\odot}$ of Section 2 until about this mass.

We showed that the numerous transient sources with high-mass black holes could be understood in terms of the bimodal distribution for forming black holes. If the envelope of a star with ZAMS mass in the range $\sim 20-35 \mathrm{M}_{\odot}$ is removed only late by common envelope evolution with a low-mass main sequence star, then the He core will burn as if clothed; i.e., like a single star. The result will be a high-mass black hole. If the common envelope occurs either during the $\mathrm{H}$ shell burning or very early supergiant phase, the two stars will merge. Thus, in our scenario the very close binaries merge, with the wider ones giving high-mass black holes. The transient outbursts arise from matter transferred onto the accretion disk of the latter by main sequence companion.

In the literature, generally a single mass limit, usually taken as $\sim 40 \mathrm{M}_{\odot}$ based on the work of van den Heuvel \& Habets (1984), is taken, ZAMS masses above this going into high-mass black holes irrespective of whether the star is single or a member of a binary. Brown, Weingartner \& Wijers (1996) made clear that this did not apply to binaries. Since binaries are necessary in gravitational merger of black holes, the fact that a much higher mass limit is required there has strong implications for gravitational merger as shown by Bethe \& Brown (1998b).

We would like to thank Stan Woosley, who not only provided us with most of the results we used, but also clarified their interpretation in several communications. We also thank Adam Burrows for a clear explanation of the consequences of skipping the convective carbon burning. We are grateful to Kip Thorne for the evaluation of our results for LIGO. We wish to thank Simon Portegies Zwart for several helpful discussions. We were supported by the U.S. Department of Energy under Grant No. DE-FG02-88ER40388. 


\section{REFERENCES}

Bailyn, C. D., Jain, R. K., Coppi, P., \& Orosz, J. A. 1998, ApJ, 499, 367

Bethe, H. A. \& Brown, G. E. 1998a, ApJ, to be published (astro-ph/9802084)

Bethe, H. A. \& Brown, G. E. 1998b, ApJ, submitted

Bodenheimer, P. \& Taam, R.E. 1984, ApJ, 280, 771

Brown, G. E. 1997, Phys. Bl., 53, 671

Brown, G. E. \& Bethe, H. A. 1994, ApJ, 423, 659

Brown, G. E., Weingartner, J. C., \& Wijers, R. A. M. J. 1996, ApJ, 463, 297

Callanan, P.J. et al, 1996, ApJ, 461, 351

Cappellaro, E. et al. 1997, A\&A, 322, 431

Chen, W., Shrader, C. R., \& Livio, M. 1997, ApJ, 491, 312

Cordes, J.M. \& Chernoff, D.F. 1997, astro-ph/9707308

Fryer, C., \& Kalogera, V. 1997, ApJ, 489, 244

Habets, G.M.H.J. 1986, A\&A, 165, 95

Iben, I., Tutukov, A.V., \& Yungelson, L.R. 1995, ApJS, 100, 217

Li, G. Q., Lee, C.-H., \& Brown, G. E. 1997, Nucl. Phys. A, 625, 372; Phys. Rev. Lett., 79, 5214

Orosz, J. A., et al., 1998, ApJ, 499, 375

Phinney, E.S. 1991, ApJ, 380, L17

Portegies Zwart, S. F. 1998, private communication

Portegies Zwart, S. F., Verbunt, F., \& Ergma, E. 1997, A\&A, 321, 207

Portegies Zwart, S. F. \& Yungelson, L. R. 1998, A\&A, 332, 173

Prakash, M., Bombacci, I., Prakash, M., Ellis, P. J., Lattimer, J. M., \& Knorren, R. 1997, Phys. Rept., 280, 1 
Rees, M.J. 1997, astro-ph/9701162, Proceedings of 18th Texas Symposium on Relativistic Astrophysics, Chicago, IL, 15-20 Dec 1996

Ruffert, M. 1994, ApJ, 427, 351

Ruffert, M. \& Arnett, D. 1994, A\&AS, 106, 505

St.-Louis, N., et al., 1993, ApJ, 410, 342

Stairs, I. H., Arzoumanian, Z., Camilo, F., Lyne, A. G., Nice, D. J., Taylor, J. H., Thorsett, S. E., Wolszczan, A. 1998, astro-ph/9712296, ApJ submitted

Thorne, K.S. 1996, in Compact Stars in Binaries, 153, IAU, ed. J. van Paradis et al.

Thorsett, S. E., \& Chakrabarty, D. 1998, astro-ph/9803260, ApJ, submittted

Thorsson, V., Prakash, M., \& Lattimer, J. M. 1994, Nucl. Phys. A, 572, 693

Timmes, F. X., Woosley, S. E., \& Weaver, T. A. 1996, ApJ, 457, 834

van den Heuvel, E. P. J. 1994, Saas-Fee Advanced Course 22: Interacting Binaries, Springer-Verlag, eds. S.N. Shore et al.

van den Heuvel, E. P. J., \& Habets, G.M.H.J. 1994, Nature, 309, 598

van den Heuvel, E. P. J., \& Lorimer, D. R. 1996, MNRAS, 283, L37

Weaver, T. A., \& Woosley, S. E. 1993, Phys. Rept., 227, 65

Weaver, T. A., Zimmerman, G. B., \& Woosley, S. E. 1978, ApJ, 225, 1021

Wijers, R.A.M.J. 1996, Evolutionary Processes in Binary Stars, 327-344, Klawer Acad. Publ., Eds R.A.M.T. Wijers et al.

Woosley, S. E., Langer, N., \& Weaver, T. A. 1993, ApJ, 411, 823

Woosley, S. E., Langer, N., \& Weaver, T. A. 1995, ApJ, 448, 315

Woosley, S. E., \& Weaver, T. A., 1995, ApJS, 101, 181 


\section{Appendix A : PREDICTIONS FOR GRAVITATIONAL WAVE DETECTORS}

Predictions for gravitational wave detectors resulting from neutron star binary mergers have been made by extrapolation from observed neutron star binaries. We consider those of Phinney (1991) and of van den Heuvel \& Lorimer (1996) to be most reliable. In both cases a factor of 3 for beaming is introduced, and an order of magnitude increase is made for binaries of too low intensity or too short a lifetime to be seen. Phinney arrives at a galactic merger rate of $R=10^{-5} \mathrm{yr}^{-1}$, whereas van den Heuvel \& Lorimer obtain $R=8 \times 10^{-6} \mathrm{yr}^{-1}$. Given the factor $\sim 30$ increase over observed systems in order to arrive at these estimates, the excellent agreement should possibly be viewed as somewhat coincidental.

The most important system for the estimates of the rate of binary neutron star coalescences is PSR $1534+12$. The estimate of Phinney uses a distance of $0.5 \mathrm{kpc}$, whereas van den Heuvel \& Lorimer use $0.7 \mathrm{kpc}$. This distance has recently been determined by Stairs et al. (1998) to be

$$
d=1.1 \pm 0.2 \mathrm{kpc}(68 \% \text { confidence limit }) \text {. }
$$

In taking too small a Galactic volume for 1534, Phinney and van den Heuvel \& Lorimer overestimated the number density in the universe by a large factor. Correction for the larger $d$ reduces the estimated merger rate of these authors by a factor $\gtrsim 2$.

Bethe \& Brown (1998a) evolved massive binaries starting from the known supernova

rate. Assuming a binarity of 0.5 and a Salpeter index of 1.5 , they obtained $R=1 \times 10^{-5} \mathrm{yr}^{-1}$ as the galactic merger rate for binary neutron stars. However, as noted in Section 1, Brown (1997) estimated that the pulsar in about half of these systems evolved into a black hole. This latter picture received support from Fryer \& Kalogera (1997) who showed that in $1913+16$ and 1534+12 special circumstances were required in order for the pulsar to avoid going into a black hole in the evolution of the binary. Stars with ZAMS masses $\lesssim 15 \mathrm{M}_{\odot}$ expand in He shell burning. In most cases the pulsar will not survive the accretion from the evolution of the companion, but will go into a black hole. In accord with this, no close neutron star binaries from ZAMS masses $\sim 8-15 \mathrm{M}_{\odot}$ are seen, even though these masses are much more numerous than those in the $\sim 15-20 \mathrm{M}_{\odot}$ range which are the most likely progenitors of $1534+12$ and $1913+16$.

The Bethe \& Brown (1998a) evolution was somewhat schematic. However, it reproduced accurately the rather complete computer calculations of Portegies Zwart \& Yungelson (1998), showing that the many effects left out by Bethe \& Brown were not important. Both of these calculations were evolutionary, beginning from the known supernova rate. That these rates agreed so well with the rates obtained from observed neutron star binaries by Phinney (1991) and van den Heuvel \& Lorimer (1996) gave 
credence to both sets of calculations. Note that the use of hypercritical accretion, which cut the binary neutron star rate down by a factor of 10 (the first born neutron star going $90 \%$ of the time into a black hole) in the theoretical evolution, was necessary in order to bring about this agreement. Note also that an earlier evolution by Iben, Tutukov \& Yungelson (1995), which left out effects of kick velocities and hypercritical accretion obtained a merger rate of $R \sim 3 \times 10^{-4} \mathrm{yr}^{-1}$ for the Galaxy, nearly two orders of magnitude greater than either the above evolutionary calculations or rates derived from observed neutron-star binaries.

Let us now make an estimate of the black-hole contribution to mergers of compact objects giving rise to gravitational waves. Since the estimates of the rate for LIGO were made as the basis of Phinney's $R=10^{-5} \mathrm{yr}^{-1}$ for the Galaxy, we also begin from this rate, arrived at by Bethe \& Brown (1998a). As noted (Brown 1997) we believe that about half of the binaries counted as double neutron stars, actually end up as black-hole, neutron-star for binaries after expansion of the companion in He shell burning has transferred matter to the pulsar. The He envelope is not very massive, $\sim 0.7 \mathrm{M}_{\odot}$ (Habets 1986; for a $2.5 \mathrm{M}_{\odot}$ He star) and, scaling crudely from Habets' calculation of envelope transfer during ${ }^{12} C$ shell burning to a $17 \mathrm{M}_{\odot}$ hydrogen star companion at a separation of $84 \mathrm{M}_{\odot}$, we estimate that half of the envelope would be transferred to the pulsar resulting from the explosion of the first He star in a He-star binary. More detailed calculation by Fryer \& Kalogera (1997) find that when the companion He star expands in He and C shell burning, the pulsar usually goes into a black hole; they adduce special circumstances to explain the survival of 1913 and 1534. We estimate that for most stars of ZAMS masses $\lesssim 15 \mathrm{M}_{\odot}$ the He core expands in red supergiant phase and engulfs the pulsar. This comprises $\sim 50 \%$ of the original double helium-star progenitors. This is just an estimate because detailed calculation to determine this fraction have not be carried out.

The combination of masses that will be well determined by LIGO is the chirp mass:

$$
M_{\text {chirp }}=\mu^{3 / 5} M^{2 / 5}=\frac{\left(M_{1} M_{2}\right)^{3 / 5}}{\left(M_{1}+M_{2}\right)^{1 / 5}}
$$

where $\mu$ is the reduced mass of the binary and $M$ is the total system mass. Taking for simplicity a circular orbit, the rate at which the frequency sweeps or "chirps" $d f / d t$ (or equivalently the number of cycles spent near a given frequency $n=f^{2}(d f / d t)^{-1}$ ) is determined solely by the chirp mass of the binary (Thorne 1996). The sensitivity of the detector is best in the $\sim 100 \mathrm{~Hz}$ range, well before actual merger, when Newtonian dynamics should be adequate.

The chirp mass of a neutron star binary, with both neutron stars of mass $1.4 \mathrm{M}_{\odot}$, is

$$
M_{\text {chirp }} \simeq 1.2 \mathrm{M}_{\odot} \quad(\mathrm{NS} / \mathrm{NS} \text { binary })
$$


The chirp mass corresponding to the Bethe \& Brown (1998a) low-mass black-hole, neutron-star binary, with mass $2.4 \mathrm{M}_{\odot}$ and $1.2 \mathrm{M}_{\odot}$, respectively,

$$
M_{\text {chirp }} \simeq 1.6 \mathrm{M}_{\odot} \quad(\mathrm{LMBH} / \mathrm{NS} \text { binary }) .
$$

With our above rough estimate for the black-hole, neutron-star binary in which the black hole resulted from the pulsar by accretion from the companion He star as it expanded in He and $\mathrm{C}$ shell burning, with masses of $1.7 \mathrm{M}_{\odot}$ for the black hole and $1.4 \mathrm{M}_{\odot}$ for the neutron star,

$$
M_{\text {chirp }} \simeq 1.34 \mathrm{M}_{\odot}
$$

Since the neutron star mass could well be less than $1.4 \mathrm{M}_{\odot}$, also the black hole mass less than $1.7 \mathrm{M}_{\odot}$, this latter $M_{\text {chirp }}$ could easily be confused with that from the NS/NS binary. We thus lump the contributions eqs. (A3) \& (A5) together, since it is doubtful that the first gravitational wave search can distinguish between them. Also, the mass $1.7 \mathrm{M}_{\odot}$ for the black hole entering into eq. (A5) was rough. We, therefore, have a combined rate of

$$
R=10^{-5} \mathrm{yr}^{-1}
$$

for the Galaxy, essentially that of Phinney (1991) and van den Heuvel \& Lorimer (1996). This was the rate on which estimates for LIGO were based. Phinney's rate extended beyond our Galaxy estimates the merger rate to be

$$
3 \mathrm{yr}^{-1} \text { at } 200 \mathrm{Mpc} \text {. }
$$

We believe the work of Bethe \& Brown (1998a) gives independent support to this estimate, avoiding several assumptions that go into it.

Kip Thorne informs us that LIGO's first long gravitational-wave search in 2002-2003 is expected to see binaries with the $M_{\text {chirp }}=1.2 \mathrm{M}_{\odot}$ of eq. (A3) and $R=10^{-5} \mathrm{yr}^{-1}$ of eq. (A6) out to $25 \mathrm{Mpc}$.

Including, however, the low-mass black-hole, neutron star binaries of Bethe \& Brown (1998a) with chirp mass eq. (A4) and the high-mass black-hole, neutron-star binaries with chirp mass of $3.3 \mathrm{M}_{\odot}$ of Bethe \& Brown (1998b) gives factors of 20 and 25, respectively, over the NS/NS binary merging rate of Phinney. These factors are calculated from the signal to noise ratio which goes as $M_{\text {chirp }}^{5 / 6}$ and then cubing it to obtain the volume of detectibility, proportional to $M_{c h i r p}^{2.5}$. Thus, our inclusion of binaries containing black holes increases the NS/NS mergers by an estimated factor of 45 . We therefore predict a rate of

$$
3 \mathrm{yr}^{-1} \times\left(\frac{25}{200}\right)^{3} \times 45=\frac{1}{4} \mathrm{yr}^{-1}
$$


This rate is still slim for 2002 and 2003, although it offers a possibility already then. Enhancement of the initial LIGO interferometer planned for 2004 should reach out beyond $70 \mathrm{Mpc}$ for the rate $R=10^{-5} \mathrm{yr}^{-1}$ bringing the comfortable expected number of events as

$$
\left(\frac{70}{25}\right)^{3} \times \frac{1}{4}=5.5 \mathrm{yr}^{-1} .
$$

We thus suggest that LIGO will observe events several years earlier than the prediction based on the rate (A7), derived from $1 \times 10^{-5}$ events per year in the Galaxy. 


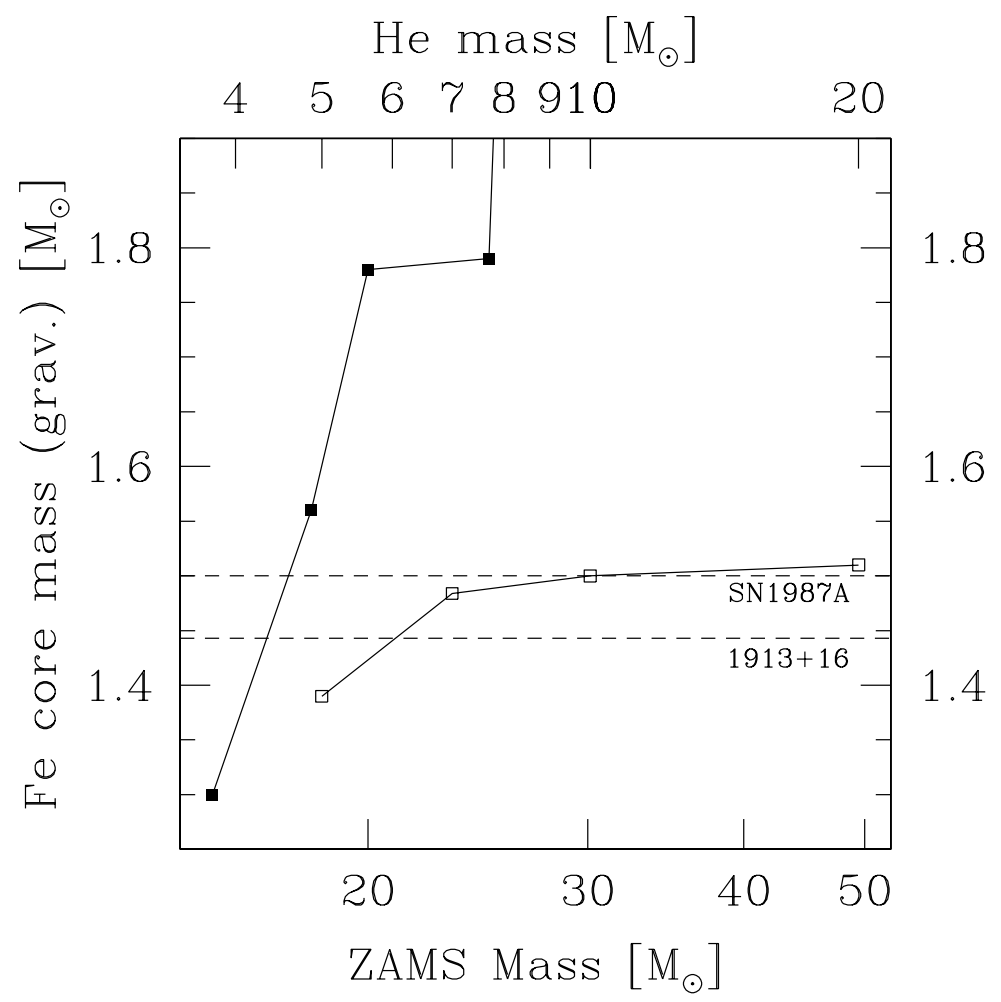

Fig. 1.- Comparison of the compact core masses resulting from the evolution of single stars (filled symbols), Case B of Woosley \& Weaver (1995), and naked helium stars (Woosley, Langer \& Weaver, 1995) with masses equal to the corresponding He core mass of single stars. The horizontal dashed lines indicate the mass of the heaviest known well-measured pulsar and the maximum mass of a neutron star. 
Table 1: Parameters of suspected black hole binaries with measured mass functions (Wijers 1996, Chen et al. 1997, Bailyn et al. 1998, Orosz et al. 1998). N means nova, XN means $\mathrm{X}$-ray nova. Numbers in parenthesis indicate errors in the last digits.

\begin{tabular}{|c|c|c|c|c|c|c|}
\hline \multirow{2}{*}{$\begin{array}{l}\text { X-ray } \\
\text { names }\end{array}$} & \multirow{2}{*}{$\begin{array}{l}\text { other } \\
\text { name(s) }\end{array}$} & \multirow{2}{*}{$\begin{array}{l}\text { compan. } \\
\text { type }\end{array}$} & $\begin{array}{l}P_{\text {orb }} \\
(\mathrm{d})\end{array}$ & $\begin{array}{l}f\left(M_{X}\right) \\
\left(\mathrm{M}_{\odot}\right)\end{array}$ & $\begin{array}{c}M_{o p t} \\
\left(\mathrm{M}_{\odot}\right)\end{array}$ & $(l, b)$ \\
\hline & & & $\begin{array}{c}K_{o p t} \\
\left(\mathrm{~km} \mathrm{~s}^{-1}\right)\end{array}$ & $\begin{array}{c}\mathrm{i} \\
\text { (degree) }\end{array}$ & $\begin{array}{c}M_{X} \\
\left(\mathrm{M}_{\odot}\right)\end{array}$ & $\begin{array}{c}d \\
(\mathrm{kpc})\end{array}$ \\
\hline $\begin{array}{l}\text { Cyg X-1 } \\
1956+350\end{array}$ & $\begin{array}{l}\text { V1357 Cyg } \\
\text { HDE } 226868\end{array}$ & O9.7Iab & $\begin{array}{c}5.5996 \\
74.7(10)\end{array}$ & $0.25(1)$ & $\begin{array}{l}33(9) \\
16(5)\end{array}$ & $\begin{array}{c}(73.1,+3.1) \\
2.5\end{array}$ \\
\hline $\begin{array}{l}\mathrm{LMC} \mathrm{X-3} \\
0538-641\end{array}$ & & B3Ve & $\begin{array}{c}1.70 \\
235(11)\end{array}$ & $2.3(3)$ & $5.6-7.8$ & $\begin{array}{c}(273.6,-32.1) \\
55\end{array}$ \\
\hline $\begin{array}{l}\text { LMC X-1 } \\
0540-697 \\
\end{array}$ & & O7-9III & $\begin{array}{c}4.22 \\
68(8) \\
\end{array}$ & $0.14(5)$ & & $\begin{array}{c}(280.2,-31.5) \\
55 \\
\end{array}$ \\
\hline $\begin{array}{l}\overline{\text { XN Mon } 75} \\
\text { A } 0620-003\end{array}$ & $\begin{array}{l}\text { V616 Mon } \\
\text { N Mon } 1917\end{array}$ & $\overline{\mathrm{K}} 4 \mathrm{~V}$ & $\begin{array}{l}0.3230 \\
443(4)\end{array}$ & $\begin{array}{c}2.91(8) \\
31-70.5\end{array}$ & $\begin{array}{c}0.2-0.7 \\
5.1-17.1\end{array}$ & $\begin{array}{c}(210.0,-6.5) \\
0.66-1.45\end{array}$ \\
\hline $\begin{array}{l}\text { XN Oph } 77 \\
\text { H } 1705-250\end{array}$ & $\mathrm{~V} 2107 \mathrm{Oph}$ & K3 V & $\begin{array}{c}0.5213 \\
420(30) \\
\end{array}$ & $\begin{array}{c}4.65(21) \\
60-80\end{array}$ & & $\begin{array}{l}(358.6,+9.1) \\
5.5:\end{array}$ \\
\hline 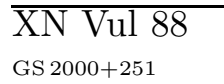 & QZ Vul & $\mathrm{K} 5 \mathrm{~V}$ & $\begin{array}{l}0.3441 \\
520(16)\end{array}$ & $\begin{array}{c}5.01(12) \\
43-74\end{array}$ & $5.3-8.2$ & $\begin{array}{c}(63.4,-3.1) \\
2\end{array}$ \\
\hline $\begin{array}{l}\text { XN Cyg } 89 \\
\text { GS } 2023+338\end{array}$ & $\begin{array}{l}\text { V404 Cyg } \\
\text { N Cyg 1938, } 1959\end{array}$ & K0 IV & $\begin{array}{c}6.4714 \\
208.5(7)\end{array}$ & $\begin{array}{l}6.07(5) \\
52-60\end{array}$ & $\begin{array}{c}0.5-1.0 \\
10-15\end{array}$ & $\begin{array}{l}(73.2,-2.2) \\
2.2-3.7\end{array}$ \\
\hline $\begin{array}{l}\text { XN Mus 91 } \\
\text { GRS 1124-683 }\end{array}$ & & $\mathrm{K} 5 \mathrm{~V}$ & $\begin{array}{l}0.4326 \\
406(7)\end{array}$ & $\begin{array}{c}3.01(15) \\
54-65\end{array}$ & $\begin{array}{c}0.56-0.87 \\
4.2-6.5\end{array}$ & $\begin{array}{c}(295.0,-6.1) \\
3.0\end{array}$ \\
\hline $\begin{array}{l}\mathrm{XN} \text { Per } 92 \\
\text { GRO J0422+32 }\end{array}$ & & M0 V & $\begin{array}{l}0.2127(7) \\
380.6(65)\end{array}$ & $\begin{array}{l}1.21(8) \\
28-45\end{array}$ & $2.6-6.9$ & $(197.3,-11.9)$ \\
\hline $\begin{array}{l}\overline{X N} \text { Sco } 94 \\
\text { GRO J1655-40 }\end{array}$ & & F5-G2 & $\begin{array}{c}2.6127(8) \\
227(2)\end{array}$ & $\begin{array}{l}3.24(9) \\
67-71\end{array}$ & $3.8-5.3$ & $\begin{array}{c}(345.0,+2.2) \\
3.2\end{array}$ \\
\hline $\begin{array}{l}\mathrm{XN} \\
4 \mathrm{U} 1543-47 \\
\end{array}$ & MX 1543-475 & $\mathrm{A} 2 \mathrm{~V}$ & $\begin{array}{c}1.123(8) \\
124(4) \\
\end{array}$ & $\begin{array}{l}0.22(2) \\
24-36 \\
\end{array}$ & $\begin{array}{l}2.3-2.6 \\
2.7-7.5 \\
\end{array}$ & $\begin{array}{c}(330.9,+5.4) \\
9.1(11) \\
\end{array}$ \\
\hline $\begin{array}{l}\overline{\mathrm{XN}} \\
3 \mathrm{U} 0042+32\end{array}$ & 2 2A $0042+323$ & G? & 11.6 & & & $\begin{array}{c}(121.3,-29.8) \\
7 ?\end{array}$ \\
\hline $\begin{array}{l}\text { XN Nor X-1 } \\
4 \mathrm{U} 1630-47\end{array}$ & $\begin{array}{l}\text { GX } 337+00 \\
\text { A1630-472 }\end{array}$ & & $\sim 1$ & & & $\begin{array}{c}(336.9,+0.3) \\
10 ?\end{array}$ \\
\hline $\begin{array}{l}\text { XN Vel } 93 \\
\text { GRS J1009-45 }\end{array}$ & & & & & & $\begin{array}{c}(276.2,+9.0) \\
3 ?\end{array}$ \\
\hline $\begin{array}{l}\mathrm{XN} \text { Cen } 67 \\
\mathrm{GS} 1354-64\end{array}$ & $\begin{array}{l}\text { Cen X-2 } \\
\text { MX1353-644 }\end{array}$ & & & & & $\begin{array}{c}(310.0,-2.8) \\
10 ?\end{array}$ \\
\hline $\begin{array}{l}\mathrm{XN} \\
\mathrm{A} 1524-62\end{array}$ & $\begin{array}{l}\text { TrA X-1 } \\
\text { KY TrA }\end{array}$ & & & & & $\begin{array}{c}(320.3,-4.4) \\
4.4\end{array}$ \\
\hline $\begin{array}{l}\text { XN Oph } 93 \\
\text { GRS } 1716-249\end{array}$ & GRO J1719-24 & & & & & $\begin{array}{l}(0.1,+7.0) \\
2.4\end{array}$ \\
\hline $\begin{array}{l}\text { XN } \\
\text { EXO 1846-031 }\end{array}$ & & & & & & $\begin{array}{c}(30.0,-0.9) \\
7 ?\end{array}$ \\
\hline $\begin{array}{l}\text { XN } \\
\text { GRS 1915+105 }\end{array}$ & & & & & & $\begin{array}{c}(45.4,-0.2) \\
12.5\end{array}$ \\
\hline $\begin{array}{l}\mathrm{XN} \\
4 \mathrm{U} 1918+146\end{array}$ & $\begin{array}{l}\mathrm{A} 1918+14 \\
\mathrm{H} 1922+154 \\
\end{array}$ & & & & & $(49.3,+0.4)$ \\
\hline
\end{tabular}


Table 2: Projected outcome for stars of various ZAMS masses

\begin{tabular}{lll}
\hline ZAMS Mass Range $\left(\mathrm{M}_{\odot}\right)$ & Single Star Fate & Fate of Star in Binary \\
\hline $0-8$ & WD & WD \\
$8-10$ & Probably NS, Possibly WD & Probably NS, Possibly WD \\
$10-18$ & NS & NS \\
$18-20$ & LMBH & NS \\
$20-35$ & HMBH & NS ? \\
$35-80 *$ & NS, LMBH & NS, LMBH \\
$80-100$ & HMBH & HMBH \\
\hline
\end{tabular}

* Note that single stars in this mass range, because of rapid mass loss, evolve similarly to naked He stars in binaries. In both cases, the compact objects are $\sim 1.5 \mathrm{M}_{\odot}$ as can be seen from Fig. 1. This mass is just our upper limit on the NS mass. 